\title{
3D Reconstruction of Brain Tumors from Endoscopic and Ultrasound Images
}

\author{
Ruben Machucho-Cadena and Eduardo Bayro-Corrochano \\ CINVESTAV, Unidad Guadalajara, \\ Department of Electrical Engineering and Computer Science \\ Jalisco, México \\ rmachuch,edb@gdl.cinvestav.mx
}

\section{Introduction}

\subsection{Statement of the Problem}

Endo-neuro-sonography (ENS) has gained special attention in recent years. Its major advantages over traditional brain surgery are that it is a minimally invasive surgical technique and the endoscopic camera and ultrasound images provide useful information. Ultrasound images are inexpensive compared to tomographic and resonance magnetic images (which are very hard to obtain in an intraoperative setting) and allow surgeons to see beyond the tissues within the brain. Another way would be to extract three-dimensional (3D) information from the combined endoscopic and ultrasound images to help surgeons better locate brain structures (such as tumors). Some work has been done in this direction, mainly in the replacement of classic ultrasound (2D imaging methodology) by 3D ultrasound equipment (Unsgaard et al., 2006). We have focused our attention on using classic ultrasound techniques and endoscopic images to extract 3D information. We propose tracking the ultrasound probe in the endoscopic images and then computing the ultrasound probe's pose in 3D space without an external method (optical or magnetic). We tested two alternative methods to track the ultrasound probe in endoscopic camera images as well as two methods to segment brain structures in ultrasound images, and then we compared the latter two types. We used conformal geometric algebra for the necessary geometric calculations and to put the results in $3 \mathrm{D}$ space.

\subsection{Outline of Our Method}

The equipment setup is as follows: The ultrasound probe is introduced through a channel in the endoscope and is seen by the endoscopic camera. With visual tracking equipment (Polaris), we can calculate the 3D position of the endoscope tip; we want to know the ultrasound (US) probe's pose in order to have the exact location of the US sensor. This is important because the US probe is flexible and rotate on its own axis. It can also move back and forth, and since the channel is wider, there is also random movement around the channel (Fig. 1). The US probe is connected to a drive unit for a micro-tip transducer; the transducer is rotated to generate a $360^{\circ}$ beam at $10 \mathrm{MHz}$. By tracking the US probe in the 
endoscopic image in successive video frames, we can use multiple-view 3D estimation techniques to find the pose of the US probe's axis. With this pose and the exact location of the endoscope's tip, we can estimate the 3D coordinates of the US probe's tip. This is fundamental since the US image is orthogonal to the US probe's axis (see Fig. 2). We know that in one small interval of time $x$, the ultrasound probe is fixed, and the endoscopic camera undergoes a movement that is equivalent to an inverse motion, that is, the endoscopic camera is fixed, and the ultrasound probe undergoes a movement. In Figs. 1 and 2, we showed the 3D virtual representation of the phantom brain used for the experiments. This model was constructed with magnetic resonance images of the phantom.

\subsection{Structure of the Chapter}

This chapter is organized as follows. Section 2 describes the techniques used to track the ultrasound probe in the endoscopic images. Two alternative methods are presented: saturation thresholding and particle filtering. Section 3 is devoted to ultrasound image processing. We present a method based on morphological operators to segment brain structures and compare its performance versus level-set methods. Section 4 describes how to calculate the 3D pose of the probe using conformal geometric algebra (CGA) and multipleview methods. We present our conclusions in Section 5.

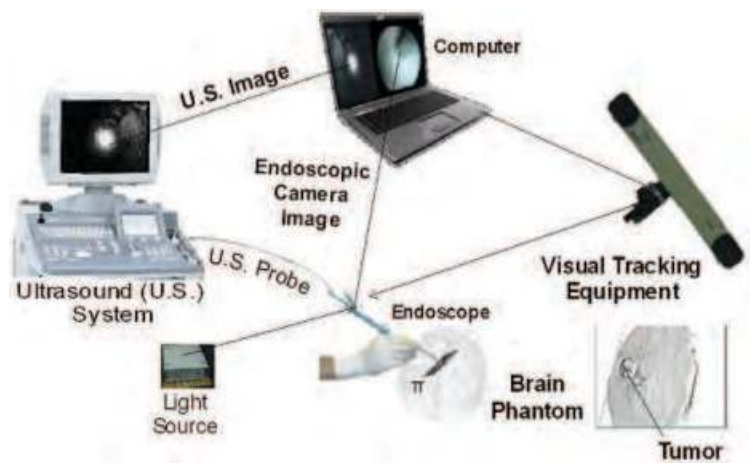




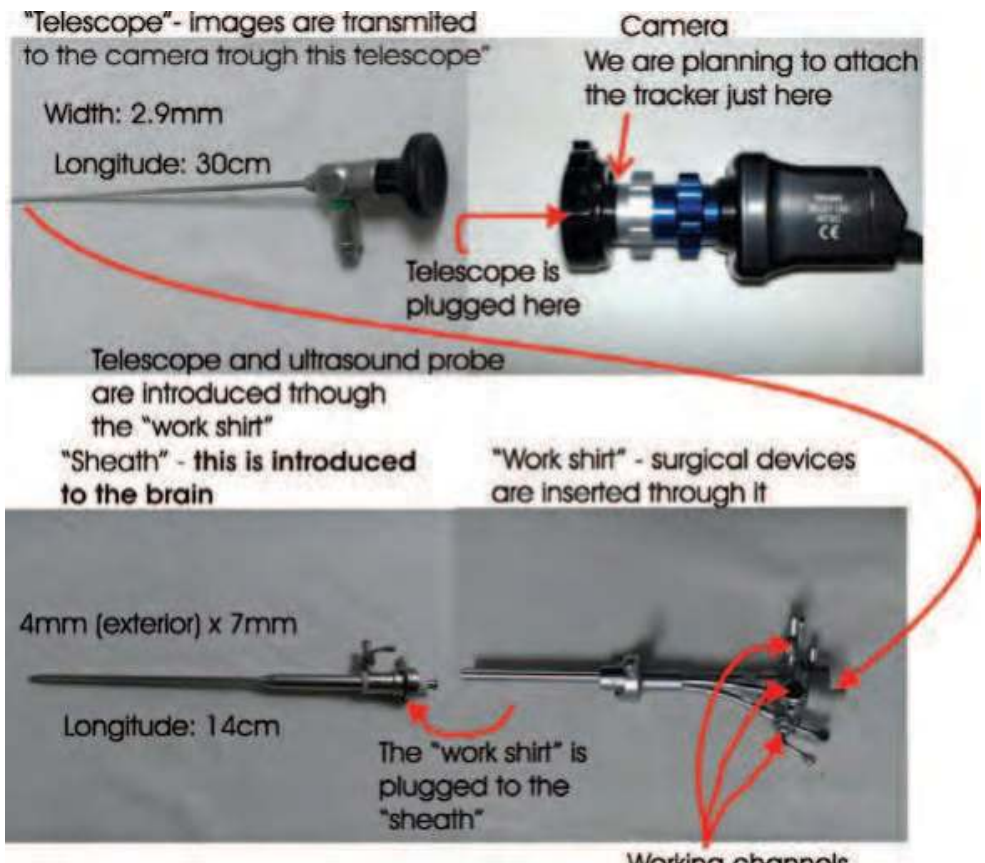

Fig. 1. ENS equipment setup.

Working channels

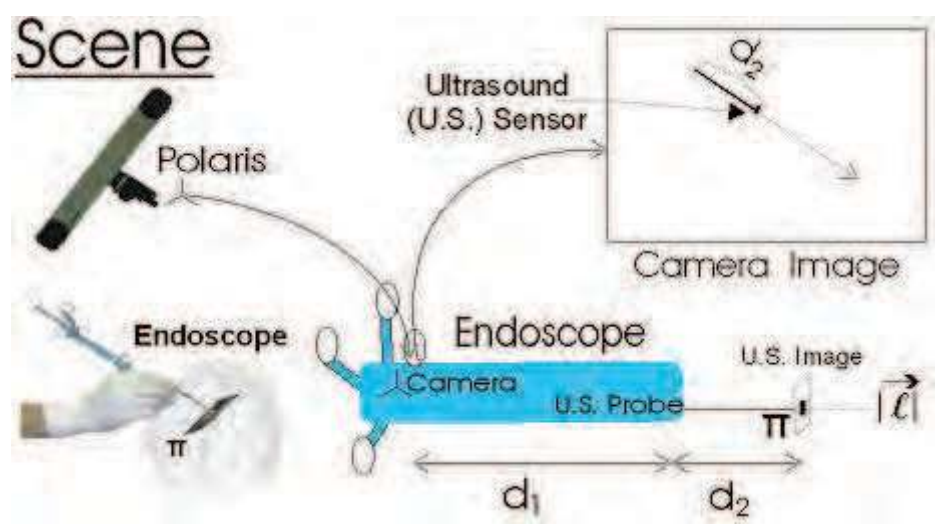

Fig. 2. Scene for the virtual representation. The plane $\pi$ that is to be calculated contains the US image to be segmented and is orthogonal to the unit vector $\ell$.

\section{Endoscopic Image Processing}

\subsection{Tracking the Ultrasound Probe}

The goal here is tracking the US probe that is seen in the endoscopic camera images. To achieve this, we use two alternative techniques: saturation thresholding and particle filters. The goal is to track the axis line of the US probe throughout the images. Knowing this line is 
important because it allows us to compute its 3D coordinates using the camera's projection matrix. Such a projection is done as follows: Every line is backprojected to form a plane in the space, which contains the line and the camera's center. Using two consecutive images, the intersection of its respective planes will yield the line in 3D (Hartley \& Zisserman, 2004). To obtain a more accurate result, we take only the unitary vector of this line (because the calculation of the translation in the projection matrix is up to a scalar factor). We translate the first point (the tip of the endoscope) obtained by the Polaris lecture by a distance $d_{2}$ (see Fig. 2) along the direction of the unitary vector in the direction of the previously obtained line, and this translated point will be the position of the US sensor in 3D, making it possible to obtain the plane $\pi$ to make the virtual representation. The $d_{2}$ distance is taken from two retroprojected points of the US probe in the 3D space, these points are begin and end of the tracked US probe in each pair of images. Polaris gives us the linear transformation between its frame and the attached tracker to the endoscopic camera and, to calculate the linear transformation between the tracker and the image frame, we use the hand-eye calibration method (Bayro-Corrochano \& Daniilidis, 1996). Now we give a brief overview of the saturation thresholding and particle filter methods that were independently used to track the axis of the US probe.

\subsection{Saturation Thresholding}

The saturation thresholding method can help segment objects like ultrasound probes. Images are converted from RGB (Red, Green, Blue) format to HSV (Hue, Saturation, Value). $V=\max (R, G, B) ; S=255^{*}(V-\min (R, G, B)) / V$ if $V \neq 0$; otherwise, $S=0$. Figure 3(a) and (b) show the saturation histogram and saturation image, respectively. Selecting all values inside [58, 255] yields the binary image in Fig. 3(c). We use the chain code to calculate the smallest and largest areas that should be eliminated, as shown in Fig. 3(d). The ultrasound probe can be described by two line segments indicating its contour. The main axis is calculated as the average segment. We cast rays from the region support endpoints and select the two rays that best encompass the segmented region [see Fig. 4(a)]. A good candidate is the ray containing more background pixels than segmented pixels. The results are shown in Fig. 4(b), which displays the main axis of the ultrasound probe. 


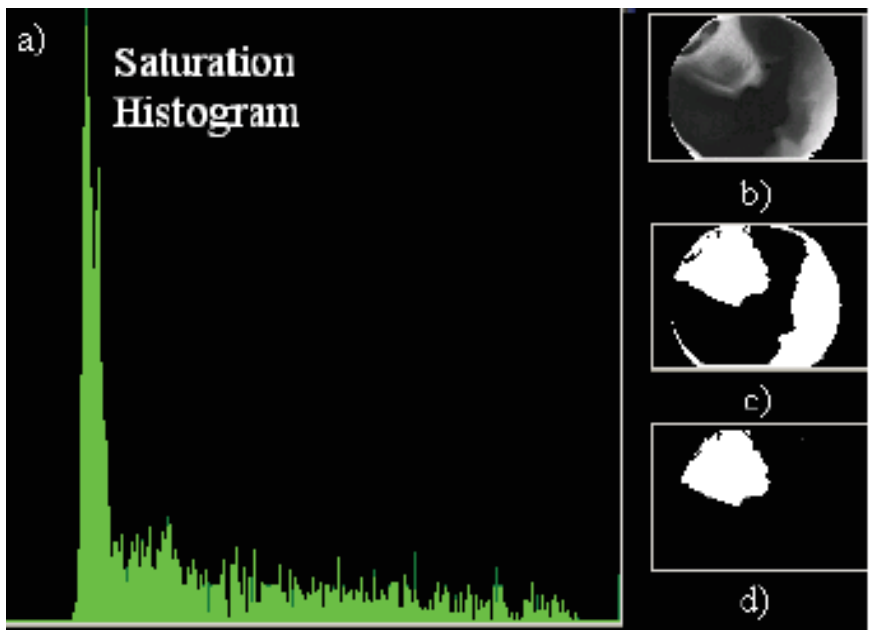

Fig. 3. Saturation histogram.

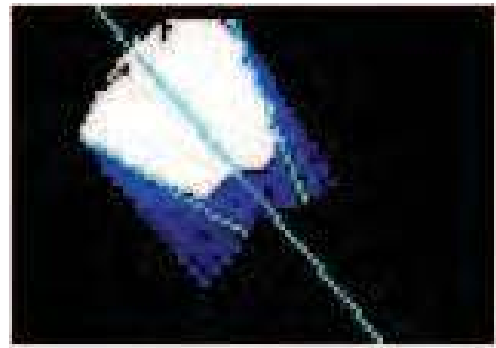

(a)

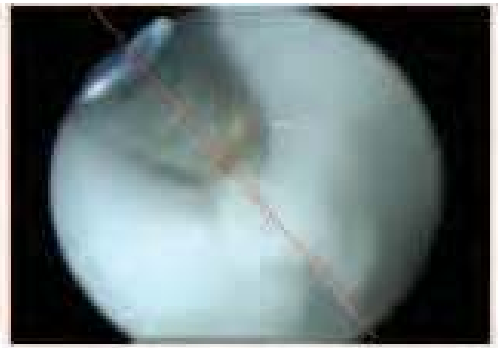

(b)

Fig. 4. Estimated line using saturation thresholding.

\subsection{Tracking with Particle Filters}

Another approach to perform visual tracking is to use a Bayesian tracker, thus treating motion tracking as a Bayesian state-estimation problem. In order to use a Bayesian framework, one must model the object being tracked as a state vector. Also needed is a method to evaluate how well the predicted states of the state vector fit the observation. The most widely used Bayesian tracker is the Kalman filter. However, Kalman filters require a Gaussian observation probability and a Gaussian posterior probability density. Our observations show that the random movement of the ultrasound probe can hardly be described as Gaussian. This situation made us look for a more general tracker: the particle filter.

\subsubsection{The Particle Filter}

Particle filters emerged from the pioneer work of Isard and Blake (1998). These filters were introduced to track objects in visual clutter and can handle multimodal observation probabilities. 
Let's assume that $x_{t}$ represents the state (state vector) of the object at time $t$ and that $X_{t}=\left\{x_{1}, \ldots, x_{t}\right\}$ represents its history over time. The vector $Z_{t}=\left\{z_{1}, \ldots, z_{t}\right\}$ encloses all the observations $z_{i}$ up to time $t$. In our framework, $z_{t}$ represents an endoscopic image at time $t$. The particle filter approximates the posterior $p\left(x_{t} \mid Z_{t}\right)$ of the probability distribution. The key idea in particle filtering is to approximate the probability distribution (and consequently the posterior) by a weighted finite set of samples, the particles. Let $S=\left\{\left(s_{t}^{(n)}, \pi_{t}^{(n)}\right) \mid n=1, \ldots, N\right\}$ be a weighted set of $N$ different samples. Every sample $s^{(i)}$ represents a possible object state, and a weight $\pi^{(i)}$ is associated with it. This weight represents the likelihood for the associated particle to be the true location of the target object. The weights are normalized so that $\sum_{i=1}^{N} \pi^{(n)}=1$.

By applying Bayes' law, the posterior $p\left(x_{t} \mid Z_{t}\right)$ can be recursively expressed as

$$
p\left(x_{t} \mid Z_{t}\right)=k_{t} p\left(z_{t} \mid x_{t}\right) p\left(x_{t} \mid Z_{t-1}\right)
$$

With the state vector at time $t-1$, the posterior $p\left(x_{t-1} \mid Z_{t-1}\right)$ can be obtained by marginalizing over $x_{t-1}$, making it possible to obtain the distribution $p\left(x_{t} \mid Z_{t-1}\right)$ :

$$
p\left(x_{t} \mid Z_{t-1}\right)=\int_{x_{t-1}} p\left(x_{t}, x_{t-1} \mid Z_{t-1}\right)=\int_{x_{t-1}} p\left(x_{t}, x_{t-1}\right) p\left(x_{t-1} \mid Z_{t-1}\right),
$$

where the chain rule was applied $\left[p\left(x_{t} \mid x_{t-1}\right)\right.$ is the dynamic model]. To perform the filtering operation, a new set of particles is created by selecting with replacement $N$ particles from the $N$ particles created at time $t-1$. The probability of selecting a particle $s^{(i)}$ is proportional to its normalized weight $\pi^{(i)}$. Then the new particles are updated using the system's evolution model. The new weights for the updated particles are calculated, measuring how well the object position represented by each particle fits with the observation $z_{t}$ at time $t$. After the weights are normalized, the mean state is estimated at each time by $E[S]=\sum_{n=1}^{N} \pi^{(n)} S^{(n)}$.

Subsequent locations of the probe can be represented as a rotation and translation with respect to the initial line estimate. A state vector can be represented as $s^{(i)}=\left[d_{x}^{(i)}, d_{y}^{(i)}, d_{\theta}^{(i)}\right]$, with its components describing this translation and rotation. This model evolves in each stage according to 


$$
S_{t}=S_{t-1}+N_{t}
$$

where $N_{t}$ is white Gaussian noise.

To obtain the weight of each particle, the image of the area selected in the first picture is obtained and rotated and translated according to the particle's (state vector) components. The transformed image is then compared to the observed image by means of the Bhattacharyya distance between their color histograms, as described by Nummiaro et al. (2003).

\subsection{Tracking Results}

The saturation thresholding (ST) method was applied as explained, and the particle filter was applied using up to $N=300$ particles, but little difference is observed in the results when $N>100$. Several images and sequences have been tested. Figure 5 shows the results for the particle filter method. Both tracking methods described performed well in practice. However, we prefer the ST method (Section 2.2) because it is faster and also because the particle filter method is nondeterministic. We obtained an accuracy of $94 \%$ with the ST method.
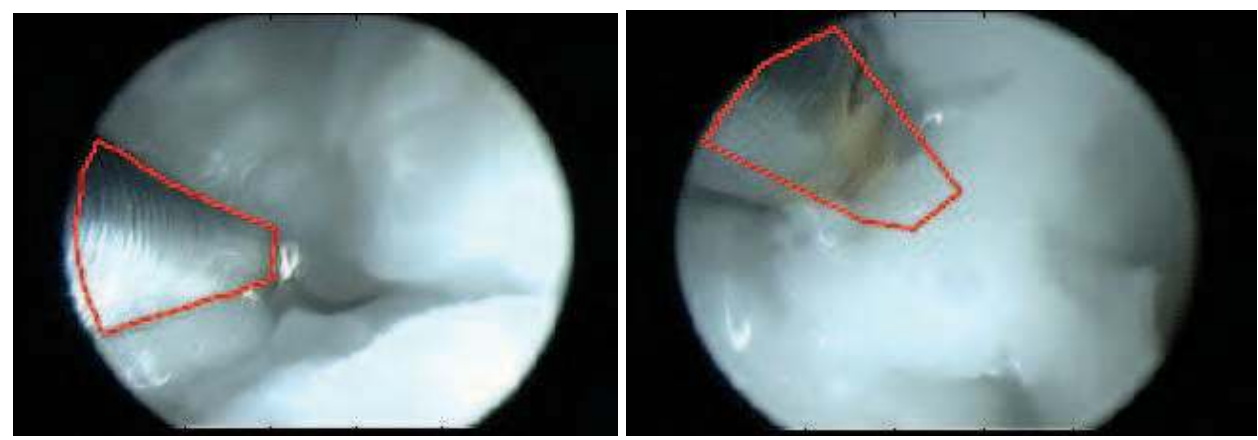

Fig. 5. The results for the particle filter method.

\section{Ultrasound Image Processing}

The goal in this stage is to segment interesting structures in the brain images, such as tumors. We used two methods in order to process the ultrasound images; the first is based on morphological operators (Castleman, 1996), and the second is the level-set method.

\subsection{Segmentation of the Tumor Using Morphological Operators}

We are using morphological operators in order to fill small holes that appear due to the subsampling provided by the Aloka ultrasound system. The closing morphological operator of image $I$ with subimage $M$ (structuring element) is defined as $I \cdot M=(I \oplus M) \otimes M$, 
where $\oplus$ and $\otimes$ represent the dilatation and erosion morphological operators, respectively.

We process the ultrasound images in the following way:

Copy the original image (do not modify it); select a region of interest (ROI); otherwise, the ROI will be the complete image. The ROI will be the same for all images.

If the ROI contains either a section of or the entire central part of the image, we exclude that part of the ROI because it only contains noise.

Normalize the ROI to minimize the contrast/brightness influence.

Apply a threshold to the gray levels of the ROI, to select only the highest levels.

Apply a closing morphological operator to fill the holes of the ROI.

Use the chain code to calculate the smallest areas of the ROI, and eliminate them.

Apply a logical AND operation between the ROI and the original image. The result is the segmented tumor, which is to be represented in $3 \mathrm{D}$.

To normalize image $I_{0}$ and to obtain $I(x, y)$, we used the following equations:

$$
\begin{aligned}
& I_{p}(x, y)=\frac{I_{0}(x, y)-\bar{I}_{0}}{\max \left(I_{0}\right)-\min \left(I_{0}\right)}, \\
& I(x, y)=255 * \frac{I_{p}-\min \left(I_{p}\right)}{\max \left(I_{p}\right)-\min \left(I_{p}\right)},
\end{aligned}
$$

where $\bar{I}_{0}$ is the mean of the image intensity and the number 255 represents the maximum gray level in the image. Figure 6 shows an example of the segmented tumor in ultrasound images.
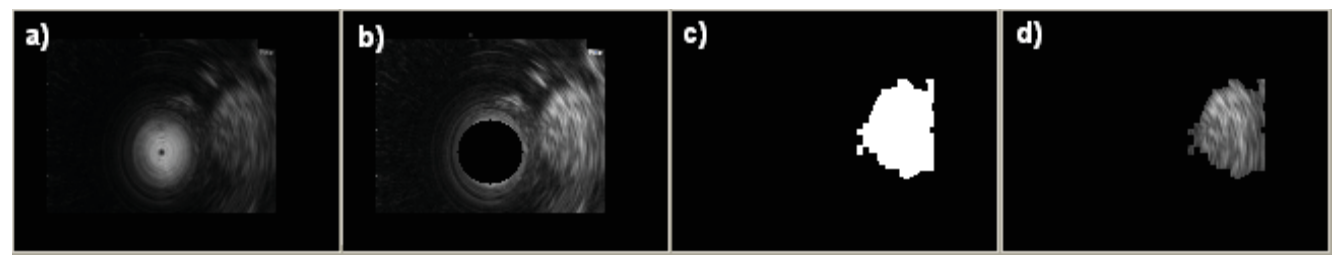

Fig. 6. Isolating the tumor. (a) Original US image to be segmented. (b) The central part of the image is excluded and the image is normalized. (c) ROI. (d) Result of segmentation.

\subsection{Segmentation of the Tumor Using the Level-Set Method}

The level-set method uses an initial seed on the image. This seed evolves with time until a zero velocity is reached or the curve has collapsed (or the maximum number of iterations is reached). To evolve the curve, the method uses two lists, called $\mathrm{L}_{\text {in }}$ and $\mathrm{L}_{\text {out }}(\mathrm{Shi}, 2005)$.

Figure 7 shows the results obtained using the level-set method. Figure 7(a) is an original ultrasound image. Figure 7(c) shows the selected and normalized ROI. Figure 7(d) shows the initial seed applied to Fig. 7(c). Figure 7(e) shows the collapsed curve. Figure 7(f) is the binary mask obtained from Fig. 7(e). This mask is applied to the original image, and we thus 
obtained the result of the segmentation [Fig. 7(b)]. The figures were obtained from Aloka ultrasound equipment using a phantom brain.
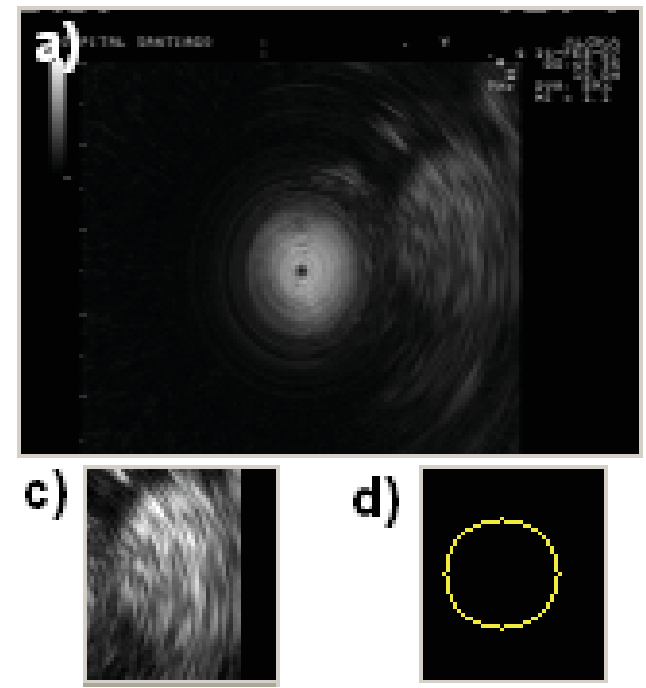

d)

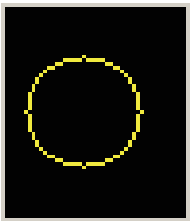

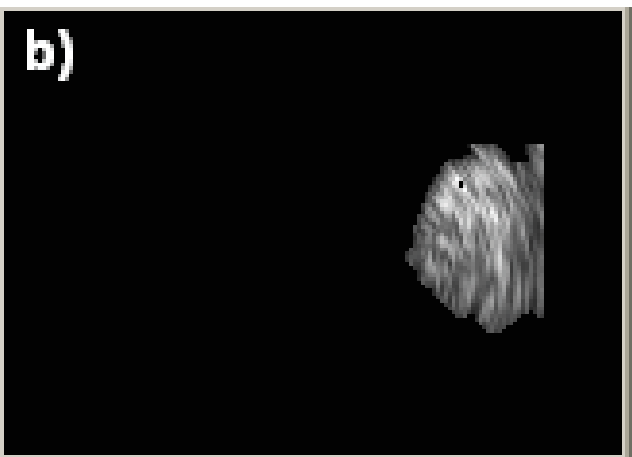

Fig. 7. Segmentation using the level-set method.

e)

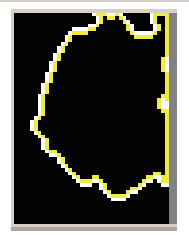

f)

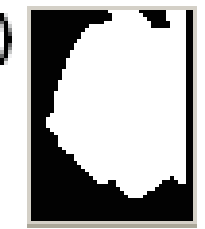

\subsection{Comparison of the Methods}

We obtained a processing time of 0.005305 seconds for the morphological operators method versus 0.009 seconds for the level-set method, that is, $188 \mathrm{fps}$ vs. $111 \mathrm{fps}$. We recommend both methods for inline implementation, because they are fast and reliable.

\section{Calculating the Ultrasound Probe's Pose}

\subsection{An Outline of Conformal Geometric Algebra}

Conformal geometric algebra (CGA) has been used in many areas such as medical image processing, robotics, and artificial vision ( $\mathrm{Li}$ et al., 2001). CGA represents geometric entities such as points, lines, planes, and spheres in an economical and compact form. CGA preserves the Euclidean metric and adds two basis vectors: $e_{+}, e_{-} ; e_{+}^{2}=1, e_{-}^{2}=-1$, which are used to define the point at the origin $e_{0}=\frac{1}{2}\left(e_{-}-e_{+}\right)$and the point at infinity $e=e_{-}+e_{+}$. The points in CGA are related to Euclidean space by $\underline{p}=p+\frac{p^{2}}{2} e+e_{0}$. A sphere in dual form is represented as the wedge of four conformal points that lie on sphere $\underline{s}^{*}=a \wedge b \wedge c \wedge d$; its

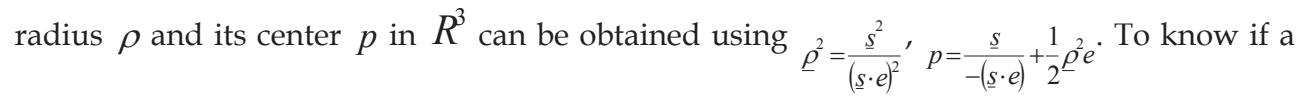
point $\underline{p}$ is into/out/on a sphere $\underline{s}^{*}$, we use the normalization $e \cdot \underline{s}=-1$ to represent the sphere as a single vector. In this way, if $\underline{p} \cdot \underline{s}>0$, then $\underline{p}$ is into the sphere; if $\underline{p} \cdot \underline{s}<0$, then $\underline{p}$ 
is out of $\underline{\pi}^{*}$; if $\underline{p} \cdot \underline{s}=0$, then $\underline{p}$ is on the sphere $\underline{\pi}^{*}$. A plane in dual form is defined as a sphere, but the last point is at infinity: $\underline{\pi}^{*}=a \wedge b \wedge c \wedge e$; the normal $\underline{n}$ to this plane is its dual $\underline{\pi}$ if the coefficient $e_{+}$of $\underline{\pi}$ is positive, else $\underline{n}=-\underline{\pi}$, so we can get the signed distance from $\underline{p}$ to $\underline{\pi}^{*}$ as $\underline{p} \cdot \underline{n}$. Figure 8 shows these concepts. A line in dual form is represented as the wedge of two points and the infinity point: $\underline{L}^{*}=a \wedge b \wedge e$. A line can also be calculated as the intersection of two planes: $\underline{L}=\underline{\pi}_{1} \wedge \underline{\pi}_{2}$. This equation is used to calculate the 3D line that represents the ultrasound probe's axis. As mentioned, we are taking only the unit vector of this line. To achieve a translation by a distance $d_{2}$ from a point $\underline{p}_{1}$ in the direction of a line, we obtain $\underline{p}_{2}: T=\exp \left(\frac{1}{2} d_{2} \underline{L}\right), \underline{p}_{2}=T \underline{p}_{1} \tilde{T}$.

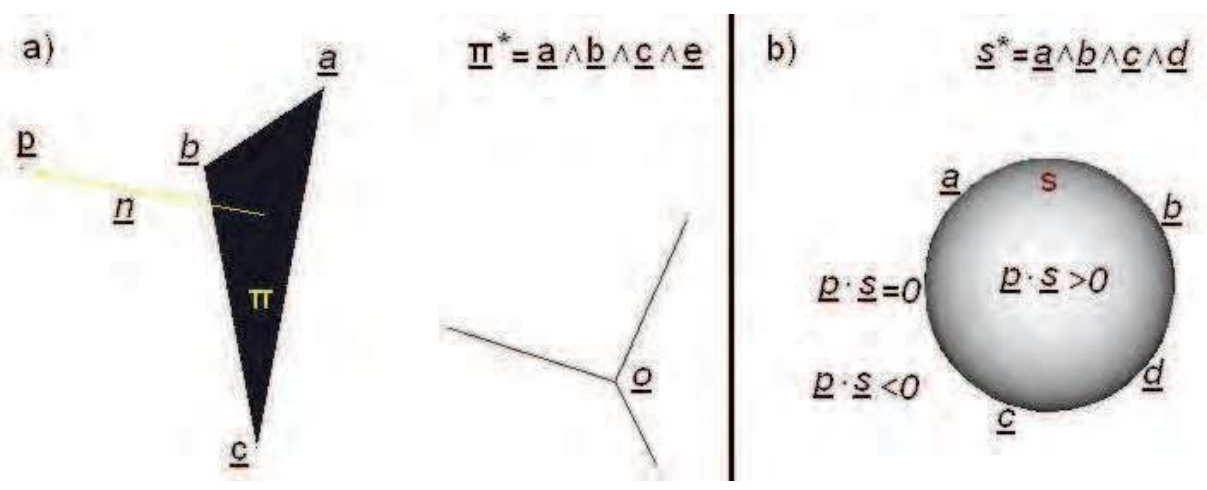

Fig. 8. Conformal entities used in the geometric tests. (a) The plane and (b) sphere.

\subsection{Putting the Results in 3D Space}

Figure 9(a) shows a slice of a tumor in 3D space; we can see the frame of the Polaris system and the calculated axis of the ultrasound probe. Figure 9(b) shows a convex hull applied to a set of slices of tumors, that is, the minimal convex set containing all the slices. This convex hull was built by using conformal geometric algebra to the geometric tests, as explained in Section 4.1. Figure 10 shows the tumor in a phantom brain model. 


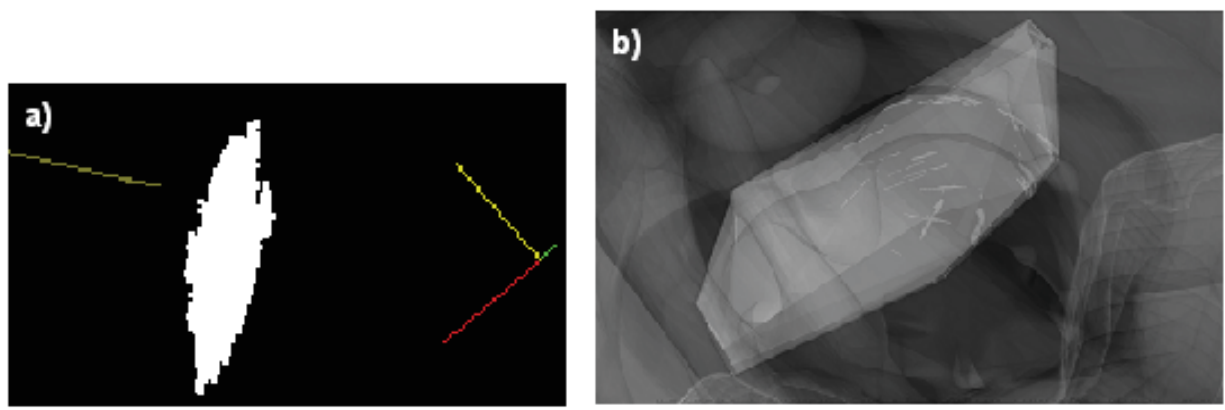

Fig. 9. (a) Virtual representation of the segmented US image. (b) Applying a convex hull to the results.

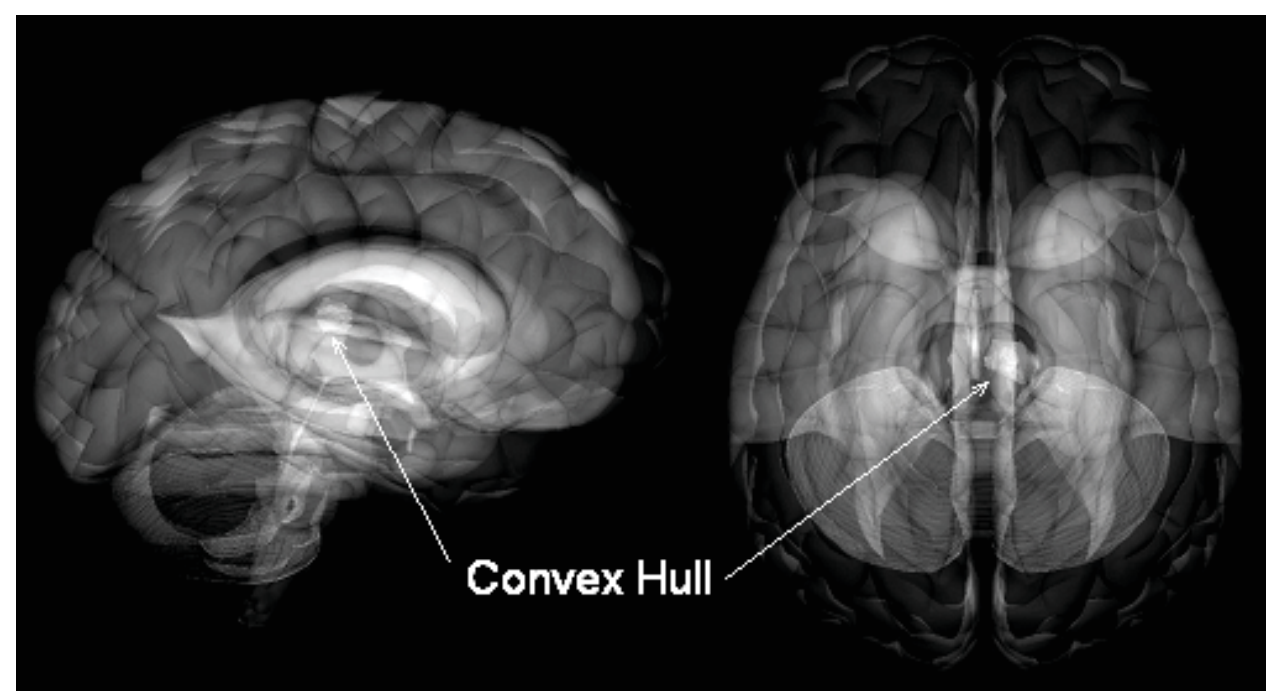

Fig. 10. The tumor in a phantom brain model.

\section{Conclusions}

In this chapter, we addressed the problem of obtaining 3D information from joint ultrasound and endoscopic images obtained with ENS equipment. In order to register both sources, we developed two alternative methods to locate the US probe's tip in endoscopic images: using saturation thresholding and a particle filter. Some preliminary results were shown. As for the ultrasound image, we presented two methods to segment interesting brain structures: morphological operators and level sets. In order to find the better method to track the ultrasound probe in the endoscopic camera images and to segment the tumor in the ultrasound images, we compared the results obtained with both methods. The results were shown in 3D space; the 3D information was calculated from the results obtained by the tracking process in endoscopic images. 
The performance of the proposed approach was demonstrated using several images that were subject to occlusions and changes in illumination and contrast. The results indicated that the proposed approach is robust.

\section{References}

Bayro-Corrochano, E.; Daniilidis, K. 1996. The dual quaternion approach to hand-eye calibration. In International Conference on Pattern Recognition (ICPR '96), Vienna, Austria, Vol. 1, pp. 318-322. IEEE Computer Society.

Castleman, K. 1996. Digital Image Processing, Prentice Hall, Englewood Cliffs, NJ.

Hartley, R.I.; Zisserman, A. 2004. Multiple View Geometry in Computer Vision, 2nd ed., Cambridge University Press, New York.

Isard, M.; Blake, A. 1998. CONDENSATION-Conditional density propagation for visual tracking. International Journal of Computer Vision, 29:5-28.

Li. H.; Hestenes, D.; Rockwood, A. 2001. Geometric Computing with Clifford Algebras: Theoretical Foundations and Applications in Computer Vision and Robotics, Springer-Verlag, New York, 2001.

Nummiaro, K.; Koller-Meier, E.; Van Gool, L. 2003. An adaptive color-based particle filter. Image and Vision Computing, 21(1):99-110.

Shi, Y. 2005. Object-based dynamic imaging with level set methods. Ph.D. thesis, Boston University.

Unsgaard, G.; Rygh, M.; Selbekk, T.; Müller, T.; Kolstad, F.; Lindseth, F.; Nagelhus Hernes, T. 2006. Intra-operative 3D ultrasound in neurosurgery. Acta Neurochirurgica, 148(3):235-253. 


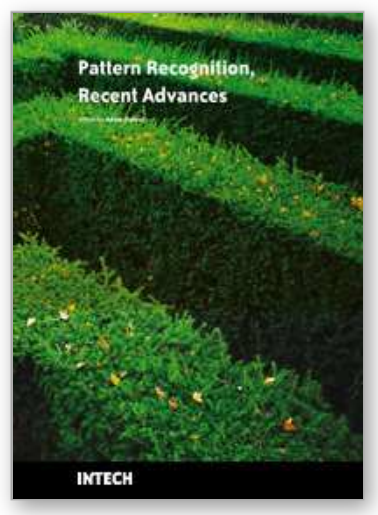

\section{Pattern Recognition Recent Advances}

Edited by Adam Herout

ISBN 978-953-7619-90-9

Hard cover, 524 pages

Publisher InTech

Published online 01, February, 2010

Published in print edition February, 2010

Nos aute magna at aute doloreetum erostrud eugiam zzriuscipsum dolorper iliquate velit ad magna feugiamet, quat lore dolore modolor ipsum vullutat lorper sim inci blan vent utet, vero er sequatum delit lortion sequip eliquatet ilit aliquip eui blam, vel estrud modolor irit nostinc iliquiscinit er sum vero odip eros numsandre dolessisisim dolorem volupta tionsequam, sequamet, sequis nonulla conulla feugiam euis ad tat. Igna feugiam et ametuercil enim dolore commy numsandiam, sed te con hendit iuscidunt wis nonse volenis molorer suscip er illan essit ea feugue do dunt utetum vercili quamcon ver sequat utem zzriure modiat. Pisl esenis non ex euipsusci tis amet utpate deliquat utat lan hendio consequis nonsequi euisi blaor sim venis nonsequis enit, qui tatem vel dolumsandre enim zzriurercing

\section{How to reference}

In order to correctly reference this scholarly work, feel free to copy and paste the following:

Ruben Machucho-Cadena and Eduardo Bayro-Corrochano (2010). 3D Reconstruction of Brain Tumors from Endoscopic and Ultrasound Images, Pattern Recognition Recent Advances, Adam Herout (Ed.), ISBN: 978953-7619-90-9, InTech, Available from: http://www.intechopen.com/books/pattern-recognition-recentadvances/3d-reconstruction-of-brain-tumors-from-endoscopic-and-ultrasound-images

\section{INTECH}

open science | open minds

\section{InTech Europe}

University Campus STeP Ri

Slavka Krautzeka 83/A

51000 Rijeka, Croatia

Phone: +385 (51) 770447

Fax: +385 (51) 686166

www.intechopen.com

\section{InTech China}

Unit 405, Office Block, Hotel Equatorial Shanghai

No.65, Yan An Road (West), Shanghai, 200040, China 中国上海市延安西路65号上海国际贵都大饭店办公楼 405 单元

Phone: +86-21-62489820

Fax: $+86-21-62489821$ 
(C) 2010 The Author(s). Licensee IntechOpen. This chapter is distributed under the terms of the Creative Commons Attribution-NonCommercialShareAlike-3.0 License, which permits use, distribution and reproduction for non-commercial purposes, provided the original is properly cited and derivative works building on this content are distributed under the same license. 